\title{
Disentangling electrons and lattice nonlinear optical response in metal-dielectric Bragg filters
}

\author{
S. Dal Conte, ${ }^{1,2}$ M. Conforti, ${ }^{3,4}$ D. Petti, ${ }^{5}$ E. Albisetti, ${ }^{5}$ S. Longhi, ${ }^{2,1}$ R. Bertacco, ${ }^{5}$ C. De Angelis, ${ }^{3}$ \\ G. Cerullo, ${ }^{2,1}$ and G. Della Valle, ${ }^{2, *}$ \\ ${ }^{1}$ Istituto di Fotonica a Nanotecnologie, Consiglio Nazionale delle Ricerche, Piazza L. da Vinci 32, I-20133 Milano, Italy \\ ${ }^{2}$ Dipartimento di Fisica, Politecnico di Milano, Piazza L. da Vinci 32, I-20133 Milano, Italy \\ ${ }^{3}$ CNISM, Dipartimento di Ingegneria dell'Informazione, Università di Brescia, Via Branze 38, I-25123 Brescia, Italy \\ ${ }^{4}$ PhLAM/IRCICA CNRS-Université Lille 1, UMR 8523/USR 3380, F-59655 Villeneuve d'Ascq, France \\ ${ }^{5}$ L-NESS and CNISM, Dipartimento di Fisica, Politecnico di Milano-Polo di Como, Via Anzani 42, I-22100 Como, Italy
}

(Received 13 November 2013; revised manuscript received 10 February 2014; published 24 March 2014)

\begin{abstract}
We investigate by broadband femtosecond pump-probe spectroscopy the transient optical response of a metaldielectric Bragg filter (MDBF) made of thin layers of gold and fused silica. A quantitative comparison of the experimental results with a semiclassical model with no fitting parameters allows us to disentangle all the different contributions to the transient optical response of the MDBF, which include the ultrafast dynamics of thermalized and nonthermalized electrons as well as of lattice phonons excited in the metal layers. Furthermore, we provide a quantitative comparison of the optical response of the MDBF with that of a single gold layer under comparable excitation conditions. The results clearly show the advantages and disadvantages of the multilayer configuration in terms of all-optical-modulation capability.
\end{abstract}

DOI: 10.1103/PhysRevB.89.125122

PACS number(s): 78.47.J-, 78.66.Bz, 42.70.Qs

\section{INTRODUCTION}

During the last decades, much progress has been achieved in the control of light-matter interaction at the nanoscale thanks to the very peculiar features exhibited by metallic structures. Metal-dielectric optical structures have been reported in plenty of configurations able to tackle a broad scenario of challenging applications ranging from nanosensing to waveguiding $[1,2]$. Most of research was devoted to the exploitation of the linear optical properties of these novel materials, which are dominated by plasmonic resonances (i.e., collective-electron oscillations coupled to electromagnetic fields [3]), whose features can be engineered by shaping the metal-dielectric interfaces. Very recently, another topic of research has been opened, which is aimed at the exploration of the nonlinear optical response of metal-based optical materials [4-7]. Actually, noble metals are well known to exhibit very high optical nonlinearities, but it is only very recently that an attempt to provide a consistent model for this nonlinearity in terms of a noninstantaneous Kerr effect has been reported [8-10]. For example, the values of the third-order nonlinear susceptibility (i.e., the $\chi^{(3)}$ coefficient) of gold retrieved from the z-scan technique reported in the literature differ by up to two orders of magnitude [11-13]. In any case, the $\chi^{(3)}$ of gold in the visible turns out to be 5-6 orders of magnitude larger than in fused silica or in other common optical glasses (cf. Ref. [8] or Ref. [10] and, e.g., Ref. [14]). Such a large value has suggested novel potential applications of gold-based structures to all-optical switching.

A key issue for the route to all-optical modulation of light is the search for the best geometry capable of full exploitation of the Kerr nonlinearity in the metallic regions. Since the refractive index of noble metals at optical frequencies is dominated by the imaginary part, causing light to evanesce, noble-metal structures ought to be optically thin (i.e., thinner

\footnotetext{
*giuseppe.dellavalle@polimi.it
}

than the skin depth, of the orders of few tens of nm in gold), but a single metal layer exhibits a poor nonlinear performance in view of the too short interaction length. It is thus desirable to play with electromagnetic boundary conditions at the metal-dielectric interface and introduce resonances in order to enhance the nonlinear response at the desired operating frequencies. This can be obtained by nanostructuring the metal regions to induce formation of localized plasmon resonances, as done for example in two-dimensional metamaterials based on gold split-ring-resonators [15] or nanopillars [16]. Another approach is to exploit cavity resonances produced by stacking multilayers of metal and dielectric films, thus forming metaldielectric Bragg filters (MDBFs) [17-20]. This approach, even though being more conventional and not applicable to scale devices down to deep subwavelength confinement, offers superior reliability and allows one to access a much higher metal volume maintaining high transmission and full control of the spectral response. For these reasons, MDBFs are promising candidates for the realization of all-optical-switching devices.

The periodic variation of the refractive index in these systems gives rise to peculiar optical spectra showing stop bands surrounded by narrow transmission peaks. Under excitation with intense laser pulses, these spectra exhibit a complex temporal dynamics induced by the noninstantaneous Kerr effect in the metal [20-22]. An accurate experimental characterization of such behavior in a broad wavelength range as well as an accurate theoretical description based on $a b$ initio modeling of the $\chi^{(3)}$ is still missing. It is worth noting that the nature of the $\chi^{(3)}$ of noble metals, which is dominated by thermomodulation of the interband optical transitions, poses an issue on such a modeling in the long-wavelength range, and the results so far reported in the literature only deal with the visible spectrum or with the near-infrared (NIR) spectrum but in concomitance with plasmonic resonances, which are dominated by the Drude permittivity (i.e., intraband transitions). Also, a direct comparison with a metallic single film is of primary importance to 
quantitatively establish the advantages (or disadvantages) of the multilayer configuration. Since the MDBF geometry exploits interference effects, causing also the linear optical response to be completely different from the optical response of a single metal layer (with remarkable effects both on absorption and transmission), a clear-cut comparison between the two configurations is in order, an issue which has not been so far addressed.

In this paper we report on an experimental and theoretical study of the ultrafast nonlinear optical response of a MDBF made of thin layers of gold and silica. The whole dynamics of carrier-carrier and carrier-lattice interaction is resolved by broadband differential transmission spectroscopy with $100 \mathrm{fs}$ time resolution. The experimental data are simulated using a semiclassical model based on an extended version of the two-temperature model (TTM) [23], which has been recently demonstrated to quantitatively reproduce the ultrafast transient optical response of a thin gold film in the visible range [24]. In the present study, the same model is applied to single out all the thermalization processes which contribute to the complex transient optical response exhibited by a MDBF in the NIR. Moreover, we provide a quantitative comparison of the optical modulation performance achieved by the MDBF with respect to a single gold film under comparable pump excitation.

\section{DEVICE FABRICATION AND LINEAR OPTICAL RESPONSE}

\section{A. Device fabrication}

A multilayer structure made of $N=5 \mathrm{Au} / \mathrm{SiO}_{2}$ stacks [Fig. 1(a)] was grown on a glass substrate by magnetron sputtering in an AJA Orion 8 system with a base pressure of $1 \times 10^{-8}$ Torr. The $\mathrm{SiO}_{2}$ layers $\left(d_{D}=260 \mathrm{~nm}\right.$ thickness $)$ were deposited by a 2-inch-diameter gun with an Ar pressure of 2 mTorr and a RF power of $200 \mathrm{~W}$, while the Au layers ( $d_{M}=15 \mathrm{~nm}$ thickness) were deposited by a 1 -inch-diameter gun, with an Ar pressure of 5 mTorr and a DC power of $10 \mathrm{~W}$. The morphological characterization of the $\mathrm{SiO}_{2}$ terminated surface was performed with an atomic force microscope (VEECO Innova) in tapping mode, with 512 samples/line and $1 \mathrm{~Hz}$ frequency. Figure 1(b) shows the image of a $10 \times 10 \mu \mathrm{m}^{2}$ area: the surface presents wide islands, of about $150 \mathrm{~nm}$ diameter and height ranging from a few $\mathrm{nm}$ up to $15 \mathrm{~nm}$, so that the roughness is $3.6 \mathrm{~nm}$ (rms). As a matter of fact $\mathrm{SiO}_{2}$ deposited by magnetron sputtering, in the same conditions but on flat substrates, displays very low roughness (lower than $1 \mathrm{~nm}$ ), so that the observed sample roughness reflects the $3 \mathrm{D}$ growth of the gold films underneath. This is not surprising because it is well known from the literature that gold tends to form nanoparticles, at the early stage of growth on oxides, which then promote a 3D morphology of the film [25]. Indeed, the corrugation of the gold layers is very visible in the cross-sectional scanning electron microscope (SEM) image of Fig. 1(c), acquired with a Philips XL30 microscope in tilted configuration $\left(9^{\circ}\right)$ on the edge of a broken sample. In the SEM image of Fig. 1(c) only three periods are visible due to the exfoliation of this portion of the film during the sample breaking, which is coherent with the black shadow seen below these layers.
The thickness of the $\mathrm{SiO}_{2}$ layers appears slightly different due to presence of some terraces, again reflecting film exfoliation, and also due to drift and defocusing effects in the high-resolution acquisition mode used to collect the main image in Fig. 1(c). The high reproducibility of the $\mathrm{SiO}_{2}$ film thickness $(260 \pm 2 \mathrm{~nm})$ can be instead observed from the low-resolution SEM scan taken on another portion of the sample, and reported in the inset of Fig. 1(c).

To establish a quantitative comparison of the multilayer configuration with a single gold layer in terms of optical modulation capability (see Sec. V), we fabricated a second sample made of a single metal film grown on a $\mathrm{SiO}_{2}$ glass substrate. The metal film thickness was selected to fulfill the following requirements: (i) measurable transmission in the NIR; (ii) sizable (i.e., not too small) volume of gold, allowing efficient exploitation of the metal nonlinearity. Note that the choice of a thickness equal to the sum of all the metal layers of the MDBF, that is $75 \mathrm{~nm}$, completely misses the first requirement since transmission from a metallic layer much larger than the skin depth $\delta=\lambda /\left(2 \pi \operatorname{Im}\left\{n_{A u}\right\}\right)$ (of the order of $30 \mathrm{~nm}$ around $800 \mathrm{~nm}$ wavelength) is practically zero. Therefore we selected a thickness of $30 \mathrm{~nm}$, corresponding to two MDBF gold layers, giving rise to nonnegligible transmission (as detailed below).

\section{B. Linear optical characterization}

The linear optical response of the MDBF and of the single metal film was investigated by transmission measurements using a spectrophotometer (JASCO V-570) with 2-nm-resolution bandwidth. The results are reported in Figs. 2(a) and 2(b) for the MDBF and for the single film, respectively. Figure 2 also shows the theoretical transmission $T$, reflection $R$, and absorption $A=1-R-T$ of the two structures, computed by standard transfer matrix method (TMM) of multilayer optical media (see, e.g., Ref. [26]). For numerical computation we adopted a phenomenological Drude-Lorentz model for gold permittivity $\epsilon_{A u}(\lambda)$ allowing accurate analytical fitting of the Johnson and Christy experimental data [27]. For fused silica, we considered a purely real dispersive permittivity with 6 Sellmeier coefficients, as detailed in Ref. [28].

The experimental transmission turns out to be in good agreement with the theoretical one, both for the MDBF and for the single film, with slight quantitative discrepancy, more pronounced for the MDBF. Part of the discrepancy can arise from deviations of layer thicknesses with respect to the nominal values. As an example, we found that a $1.5 \mathrm{~nm}$ increase in the metal thickness can bridge the gap between the experimental and the theoretical transmission in the single metal layer structure, and it is expected that similar deviations can have an even higher impact on the multilayer configuration. However, in light of the results of the morphological characterization detailed in Sec. II A, most of the discrepancy is ascribable to extra losses (not considered in the TMM computation) due to scattering from defects and corrugations at the metal-dielectric boundaries or due to absorption from residual fabrication impurities. Therefore, the observed discrepancy is expected to have negligible influence on the differential transmission, since the $\Delta T / T$ rejects all the additional losses affecting the probe signal (see following sections). 

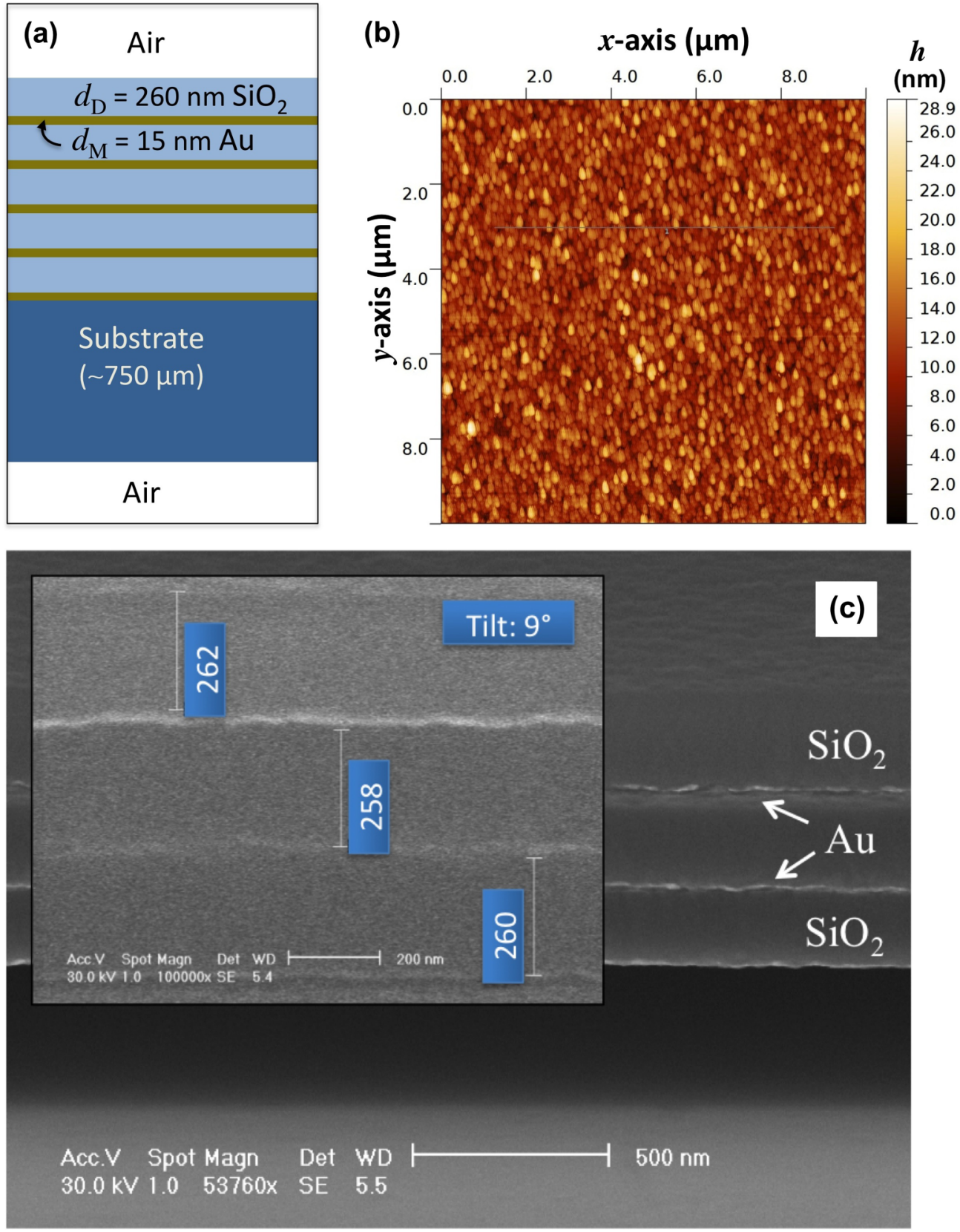

FIG. 1. (Color online) The MDBF. (a) Sketch of the multilayer geometry. (b) $10 \times 10 \mu \mathrm{m}^{2}$ AFM image of the surface of the terminating $\mathrm{SiO}_{2}$ layer. (c) Cross-sectional SEM images of the sample. In the inset a low-resolution fast scan is shown, in order to get rid of drift and defocusing effects in the evaluation of the $\mathrm{SiO}_{2}$ film thickness (numbers in the blue boxes are in $\mathrm{nm}$ ).

Note that the MDBF exhibits relatively narrow transmission and absorption peaks in the NIR, at $\sim 840, \sim 920$, and $\sim 1040 \mathrm{~nm}$, whereas the single film shows an almost flat spectrum in this same wavelength range. Conversely, below the stop band (spanning the 600-800 nm range) transmission peaks are almost completely washed out. This is ascribable to the onset of interband transitions in the metal which in concomitance with the interference effects of the multilayer configuration causes a huge increase in the absorption as compared to the single metal layer [compare dotted curves in Figs. 2(a) and 2(b)].

\section{TRANSIENT OPTICAL RESPONSE VIA PUMP-PROBE SPECTROSCOPY}

Since the Kerr nonlinearity of noble metals is noninstantaneous in nature, the most suitable experimental tool to investigate the optical modulation capability of metalbased optical structures is ultrafast pump-probe spectroscopy. Our ultrafast spectroscopy setup starts with an amplified Ti:sapphire laser (Integra-C from Quantronix) producing $100-\mathrm{fs}, 1-\mathrm{mJ}$ pulses at $800 \mathrm{~nm}$ and $1 \mathrm{kHz}$ repetition rate. The MDBF is excited by tunable $100-\mathrm{fs}$ pulses produced by an optical parametric amplifier, and the differential transmission 

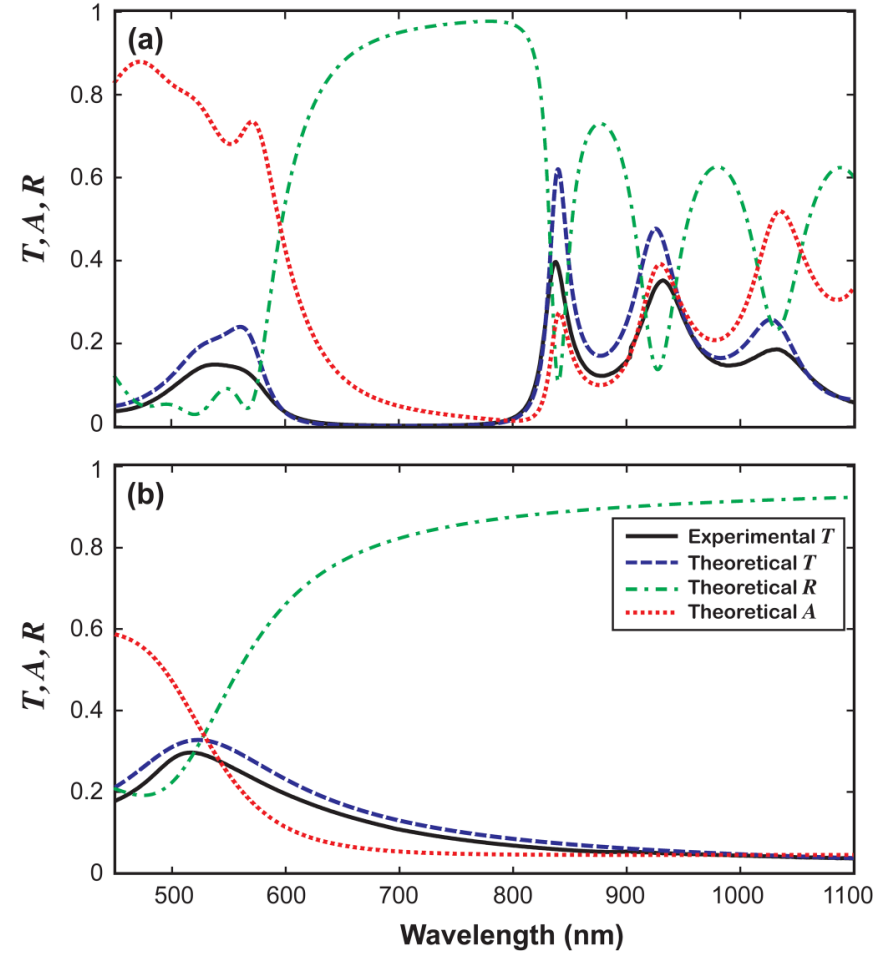

FIG. 2. (Color online) Linear optical response of (a) the MDBF and (b) a single gold layer of $30 \mathrm{~nm}$ thickness for comparison.

$(\Delta T / T)$ is probed by white light continuum pulses generated in a sapphire plate. Broadband detection is achieved with an optical multichannel analyzer (Stresing Entwicklungsbüro) working at the full $1 \mathrm{kHz}$ laser repetition rate [29]. Figure 3(a) shows a $\Delta T / T$ map, as a function of probe wavelength and pump-probe delay, for the MDBF excited at $\lambda_{P}=835 \mathrm{~nm}$, that is close to the peak of one absorption resonance in the optical spectrum of Fig. 2(a). A complex dynamics is observed, with several positive and negative peaks evolving on different time scales between $100 \mathrm{fs}$ and few ps. Experimental $\Delta T / T$ dynamics are reported in Fig. 4 (solid blue curves) for three probe wavelengths: $915 \mathrm{~nm}$ and $960 \mathrm{~nm}$, where the spectral modulation turned out to be more pronounced, and $980 \mathrm{~nm}$, which is representative of an intermediate behavior between one peak and one dip of the map.

Note that not only the $\Delta T / T$ signal peaks at different time delay for different probe wavelengths, but also sign changes are observed [Fig. 4(b)], as well as ultrafast oscillatory dynamics on the shortest time scale (before 500 fs time delay) [Fig. 4(c)]. On the longer time scale [Fig. 5(a)] the dynamics turned out to be much simpler, with the onset of an almost stationary transient spectrum that exponentially decays to zero within a few hundred ps (a time constant of $\sim 50 \pm 10 \mathrm{ps}$ for the exponential decay was estimated). Nevertheless, this longliving $\Delta T / T$ spectrum exhibits rich features with positive and negative peaks as well. Finally, note that contrary to what has been reported in metal nanoparticles and thin films no relevant oscillatory dynamics is observed on this time scale. In fact, an accurate analysis of the dynamics of Fig. 5(a) reveals the presence of a very weak oscillation superposed on the long-living signal [Fig. 5(b)]. The oscillation is dominated

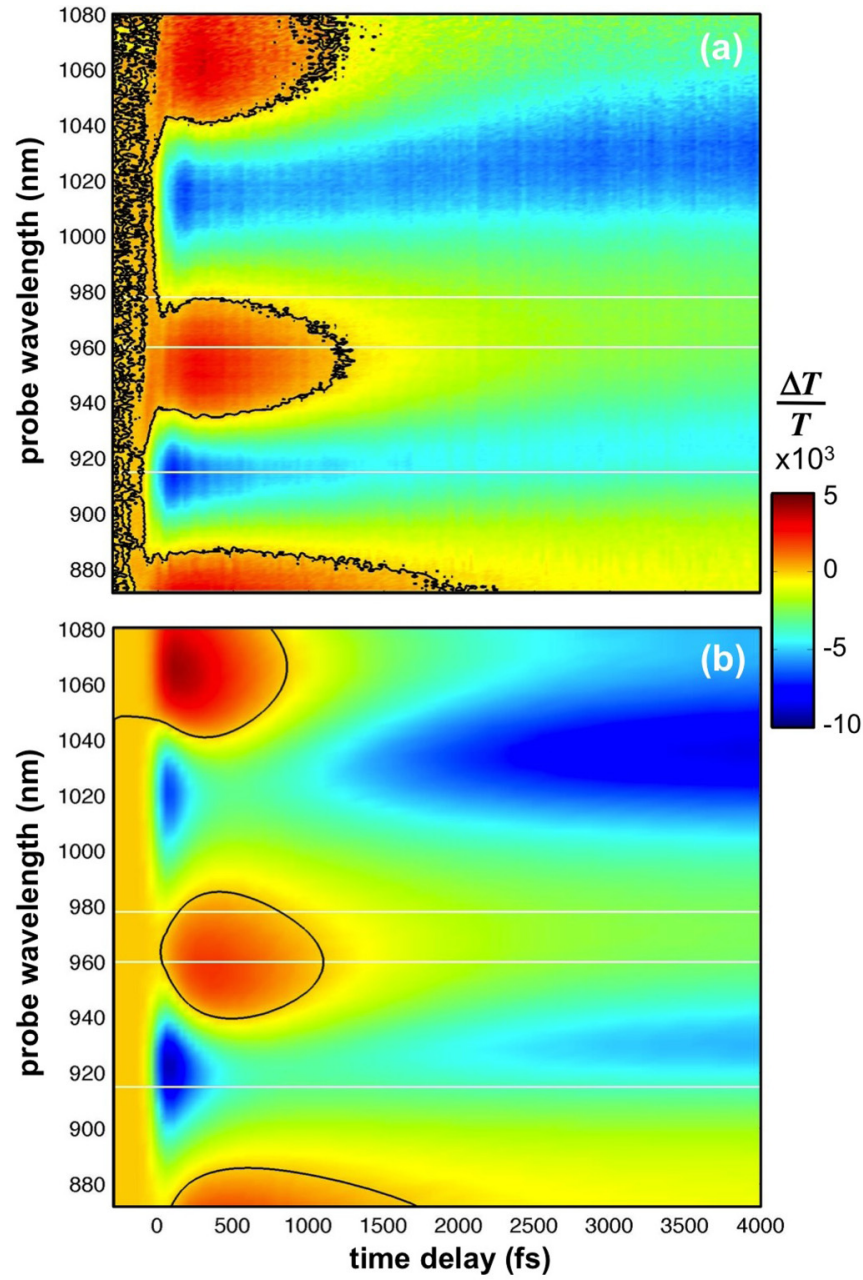

FIG. 3. (Color online) Differential transmission map of the MDBF under incident fluence $F \simeq 320 \mu \mathrm{J} / \mathrm{cm}^{2}$ : (a) experimental data; (b) numerical simulation. For simulations, we assumed $\tau_{P}=$ 115 fs [see Eq. (2)] corresponding to the resolution time of the experiment. The black curves show the isosbestic, i.e., the locus of zero in the $\Delta T / T$ maps. Thin horizontal lines correspond to the temporal cross sections reported in Fig. 4.

by a sinusoidal component with period $T_{\text {osc }} \simeq 7.2 \pm 0.5 \mathrm{ps}$ [Fig. 5(c)], that is exponentially damped with a time constant $\tau_{\mathrm{osc}} \simeq 8 \pm 3 \mathrm{ps}$.

\section{THEORETICAL MODELING AND NUMERICAL SIMULATIONS}

To quantitatively model the temporal evolution of the transient optical spectra obtained from pump-probe experiments, we followed an approach similar to the one reported in a previous work [24]. The key point of the model is the calculation of the modulation of the gold dielectric function, $\Delta \epsilon$, induced by pump absorption. Our approach allows one to segregate this computation into distinct steps, as summarized schematically in Fig. 6. Provided that the pump photon energy is below the edge of the interband optical absorption of gold (around $2.15 \mathrm{eV}$ ), the pump pulse is absorbed via the linear (i.e., single photon) excitation of the plasmon-polaritons of the metallic structure (i.e., the volume plasmon for metallic films 

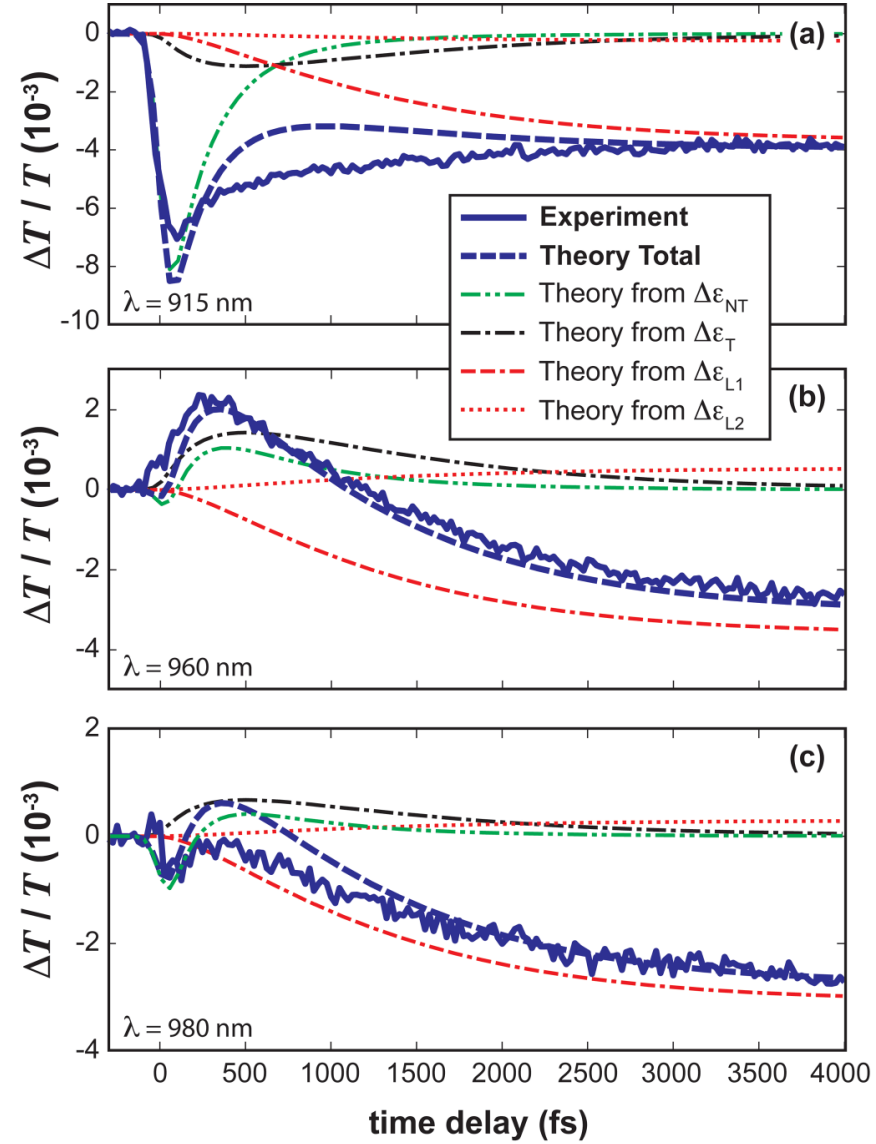

FIG. 4. (Color online) Cross sections of the $\Delta T / T$ maps of Fig. 3 at three different probe wavelengths: (a) $915 \mathrm{~nm}$, (b) $960 \mathrm{~nm}$, and (c) $980 \mathrm{~nm}$. Experimental data (solid lines) are quantitatively compared to numerical simulations showing the total $\Delta T / T$ (dashed lines) and the four different contributions to the $\Delta T / T$ arising from nonthermalized (dash-double-dot lines) and thermalized (dash-dot lines) electrons, and from the lattice, including the effect related to the Drude damping (double-dash-dot lines) and plasma frequency (dotted lines).

or the localized surface plasmon for metallic nanostructures). Within few ten fs, the plasmonic oscillation is dephased, leaving the free carrier gas in an out-of-equilibrium state with a nonthermal energy distribution. Nonthermalized electrons release their excess energy to the other part of the electronic population, that is thermalized electrons, in a few hundred fs by means of electron-electron scattering. The hot thermalized carriers, in turn, exchange energy with the colder lattice by electron-phonon scattering on the picosecond time scale.

\section{A. Electrons and lattice temporal dynamics}

The dynamical process of light absorption and subsequent relaxation and energy exchange between the different degrees of freedom of the metal can be accurately described by the Boltzmann equations (see, e.g., Refs. [30,31]). A much more simplified description, given within a fully classical picture, is provided by the so-called TTM which describes the equilibration process between the thermalized hot electrons and the phonon bath. Unfortunately this model completely
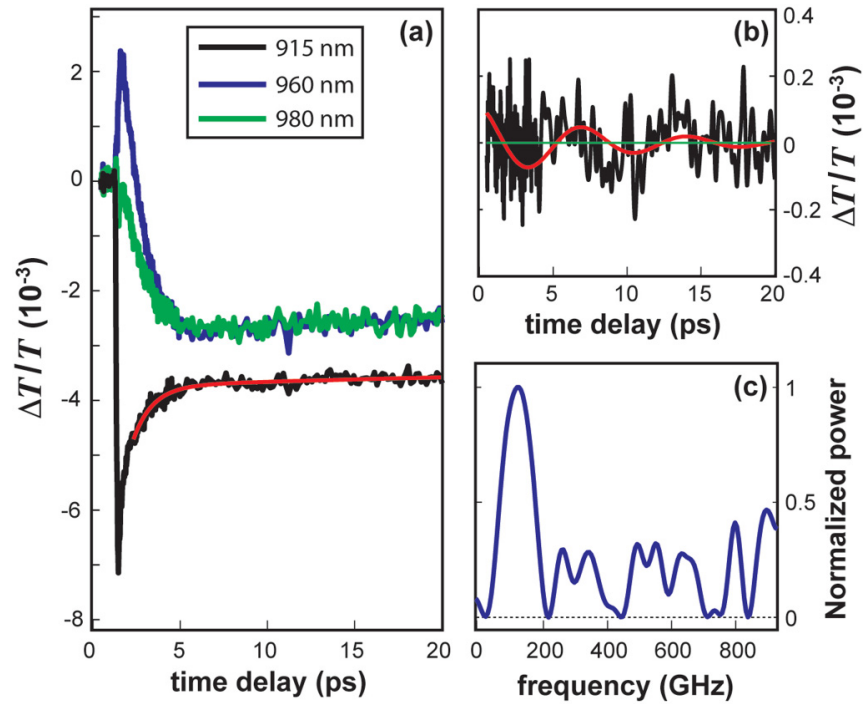

FIG. 5. (Color online) (a) Cross sections of the $\Delta T / T$ maps on a longer time scale for three different probe wavelengths. Red curve superposed on the cross section at $915 \mathrm{~nm}$ is a biexponential fitting curve of the form $C_{1} \exp \left(-t / \tau_{D 1}\right)+C_{2} \exp \left(-t / \tau_{D 2}\right)+C_{3}$. The fitting was attained starting from 1 ps in order to disregard the complex dynamics of the initial steps which is not exponential. (b) The oscillatory signal at $915 \mathrm{~nm}$ probe wavelength estimated as the difference between the actual dynamics and the biexponential fitting reported in (a). The red curve in (b) is a damped sinusoidal fitting: $A \cos \left(2 \pi / T_{\mathrm{osc}} t+\phi\right) \exp \left(-t / \tau_{\mathrm{osc}}\right)$. (c) Power spectral density of the signal in (b).

misses the dynamics of nonthermalized carriers by assuming an instantaneous thermalization of the electrons. Under the relaxation-time approximation, the Boltzmann formalism can

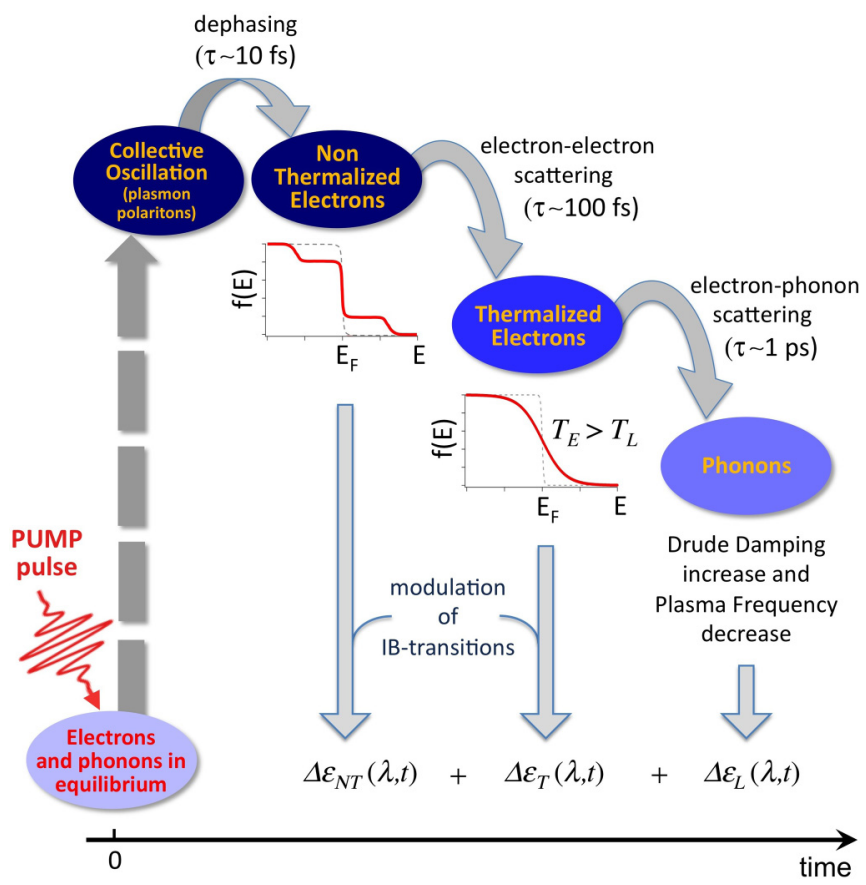

FIG. 6. (Color online) Sketch of the carrier-lattice energy exchange after pump-pulse absorption and fundamental processes considered in our model of the noninstantaneous Kerr effect in gold. 


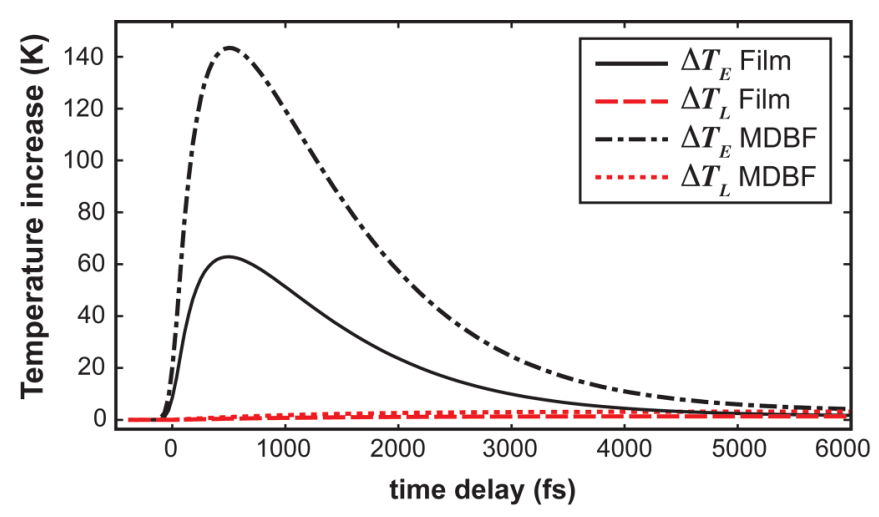

FIG. 7. (Color online) Numerical simulation of the temporal dynamics of thermalized electrons and lattice temperatures for the MDBF and for the single metal film considered in our experiments.

be recast in an extended version of the TTM (ETTM) [23] that is capable of capturing the essentials of nonthermalized carrier dynamics within a system of two coupled ordinary differential equations for thermalized electrons and lattice temperatures, $T_{E}$ and $T_{L}$.

The ETTM reads as follows:

$$
\begin{aligned}
C_{E} \frac{d T_{E}}{d t} & =-g\left(T_{E}-T_{L}\right)+\int P_{a}\left(t^{\prime}\right) H\left(t-t^{\prime}\right) d t^{\prime}, \\
C_{L} \frac{d T_{L}}{d t} & =g\left(T_{E}-T_{L}\right),
\end{aligned}
$$

where $C_{E}=\gamma T_{E}$ and $C_{L}$ are the electronic and lattice heat capacities, $g$ is the electron-phonon coupling constant, and $H(t)$ is a response function accounting for the energy transfer from nonthermalized electrons (generated by pump excitation) to thermalized electrons [32]. The explicit form of $H(t)$ and the values of the ETTM coefficients for gold can be found in Refs. [24] and [23]. In Eq. (1), $P_{a}(t)$ is the pump-pulse power absorbed in the unit volume of the metal, computed from the linear absorption of the MDBF at the pump wavelength, $A\left(\lambda_{P}\right)$ (detailed in Sec. II), according to the formula

$$
P_{a}(t)=\sqrt{\frac{4 \ln (2)}{\pi}} A\left(\lambda_{P}\right)\left(\frac{F}{N d_{M} \tau_{P}}\right) \exp \left(-\frac{4 \ln (2) t^{2}}{\tau_{P}^{2}}\right),
$$

$F$ being the incident fluence of the pump pulse and $\tau_{P}$ its time duration (FWHM).

Equation (1) is solved by numerical integration using the standard Runge-Kutta method. The resulting time-dependent electronic and lattice temperatures are reported in Fig. 7 for our case study of the MDBF and for the single film under the excitation conditions of our experiments, detailed in Sec. III.

\section{B. Spectral modeling of Kerr nonlinearity}

The spectral variation of the gold dielectric function can be computed as the sum of four different contributions (Fig. 6): two terms $\Delta \epsilon_{N T}(\lambda, t)$ and $\Delta \epsilon_{T}(\lambda, t)$ arising from nonthermalized and thermalized electrons, respectively, and two terms $\Delta \epsilon_{L 1}(\lambda, t)$ and $\Delta \epsilon_{L 2}(\lambda, t)$ arising from lattice heating according to Drude-damping increase and plasma frequency decrease mechanisms, respectively.

\section{Electronic contribution}

The contributions $\Delta \epsilon_{N T}(\lambda, t)$ and $\Delta \epsilon_{T}(\lambda, t)$ come from the modulation of the interband (IB) transition in gold caused by pump-induced temporal variations of the electron distribution accounting for nonthermalized $\Delta f_{N T}(E, t)$ and thermalized $\Delta f_{T}(E, t)$ electrons, respectively. The latter variation is given by

$$
\Delta f_{T}(E, t)=f_{0}\left[E, T_{E}(t)\right]-f_{0}\left(E, T_{0}\right),
$$

where $T_{E}(t)$ is retrieved from the ETTM, $T_{0}$ is the room temperature, $f_{0}$ is the Fermi-Dirac function, and $E$ is the electron energy. For nonthermalized carriers, similarly to Ref. [23], we have

$\Delta f_{N T}(E, t)=1 / A \times \Delta_{N T}(E) \int_{-\infty}^{t} P_{a}\left(t^{\prime}\right) e^{-\left(t-t^{\prime}\right) / \tau(E)} d t^{\prime}$,

where the explicit expressions of $\tau(E), \Delta_{N T}(E)$, and normalization constant $A$ are given in Ref. [24].

The imaginary part of the $\Delta \epsilon_{N T,(T)}(\lambda, t)$ can be computed from the $\Delta f_{N T,(T)}(E, t)$ under constant matrix element approximation once the energy distribution of the joint density of states for the considered IB transitions is determined. This can be done under parabolic band approximation from the knowledge of gold band structure as detailed in Ref. [24]. The corresponding real part of the $\Delta \epsilon_{N T,(T)}(\lambda, t)$ is then retrieved from Kramers-Kronig analysis.

Differently from previous studies [8,24], we considered here not only the main IB optical transition of gold, starting at around $2.45 \mathrm{eV}(\sim 510 \mathrm{~nm})$, involving electronic states at the $\mathrm{L}$ point in the Brillouin zone, but also the X-point IB transition, having an absorption edge at around $1.9 \mathrm{eV}(\sim 650 \mathrm{~nm})$ [33]. Even though this second IB transition is marginal for the Kerr nonlinearity in the visible [34], it could contribute to the NIR response of our experiments. Band-structure parameters (effective masses in the valence and conduction bands and energy gaps) and spectroscopical parameters (dipole matrix elements for L-point and X-point optical transitions) are the same as those reported in previous studies (see Ref. [9] and references therein). Also, we found that, as reported since the first pioneering studies on thermomodulational nonlinearity in gold films [33,34], an effective temperature of $650 \mathrm{~K}$ instead of the room temperature in the Fermi-smearing mechanism (governing the $\Delta \epsilon_{T}$ ) retrieves better agreement with the experimental data. Basically, the use of an effective temperature that is higher than the room temperature allows one to take into account the broadening of the interband transition observed in the linear absorption spectrum of gold. It is expected that this heuristic correction compensates for the hypersimplification introduced when modeling the IB optical transitions in a broad range of wavelengths, like the constant matrix element approximation and the absence of interband damping (arising from electron-phonon scattering, electron-electron Coulomb interaction, and electron scattering at the surfaces for ultrathin nanostructures). According to a recent paper by Masia and coworkers [35], the damping caused by phonon scattering of conduction electrons seems to be of the most relevance, as the broadening of the interband absorption of gold was modeled by considering the smearing of the final state induced by Drude damping (being the final 
state in the conduction band). According to this view, the broadening of the IB transition turned out to be proportional to the Drude damping $\Gamma$, with the constant of proportionality being a fitting parameter that nevertheless ought to be determined by comparison with experiments, similarly to the effective temperature parameter introduced by Rosei and colleagues.

\section{Lattice contribution}

The contribution coming from lattice heating above room temperature $T_{0}$ is related to the Drude-Sommerfeld (DS) permittivity of the metal (due to the free electrons). An increase in the lattice temperature causes an increase of the Drude damping $\Gamma$ and subsequent modification of the DS optical response. This is given by the following relation:

$$
\Delta \varepsilon_{L 1}(\lambda, t)=\left.\frac{i \omega_{P}^{2} \Delta \Gamma(t)}{\omega(\omega+i \Gamma)[\omega+i \Gamma+i \Delta \Gamma(t)]}\right|_{\omega=2 \pi c / \lambda},
$$

where

$$
\Delta \Gamma(t)=\beta\left[T_{L}(t)-T_{0}\right] \Gamma .
$$

In the above equations, $\omega_{P}=1.327 \times 10^{16} \mathrm{rad} / \mathrm{s}$ is the plasma frequency and $\Gamma=1.327 \times 10^{16} \mathrm{rad} / \mathrm{s}$ is the Drude damping of gold at room temperature [27], and $\beta=3.2 \times 10^{-3} \mathrm{~K}^{-1}$ is estimated according to Holstein's model (see, e.g., Ref. [36] and references therein).

Another effect on the DS permittivity induced by lattice heating is related to the thermal expansion of the lattice. In general, the lattice expansion can cause both a decrease of the carrier density and a variation of effective mass of the electrons [37-40]. The decrease of the free carrier density $n$ turns into a decrease of the plasma frequency, since $\omega_{P} \propto n^{1 / 2}$. In silver, an increase of lattice temperature was observed to give rise to an increase and not a decrease of the plasma frequency $[37,40]$. Therefore the variation of effective mass of electrons was recognized as the most relevant mechanism for the modulation of plasma frequency in silver. In gold, the situation is reversed, since experimental studies on gold nanoparticles indicate that an accurate quantitative interpretation of the pump-probe data can be attained by considering the carrier density mechanism only (see, e.g., Ref. [39]); i.e., the effective mass contribution can be completely disregarded in gold. We computed the contribution to the variation of gold permittivity caused by thermal expansion of the metal layers according to the equation

$$
\Delta \varepsilon_{L 2}(\lambda, t)=-\left.\frac{\Delta \omega_{P}(t)\left[\Delta \omega_{P}(t)+2 \omega_{P}\right]}{\omega(\omega+i \Gamma)}\right|_{\omega=2 \pi c / \lambda},
$$

where

$$
\Delta \omega_{P}(t)=-\frac{1}{2} \frac{\omega_{P}}{d_{M}} \Delta d_{M}(t) .
$$

In Eq. (8), $\Delta d_{M}(t)$ is the time-dependent variation of gold layers thickness (averaged on the five stacks for the MDBF) caused by lattice heating. Since the lattice is heated on a time scale of a few ps, the thermal expansion of gold layers does not follow adiabatically the lattice temperature $T_{L}(t)$. According to a standard modeling employed in metal nanoparticles and single metal films [40], the thermal expansion can be described as the excitation of acoustic resonances of the metallic nanostructure (the fundamental longitudinal acoustic resonance of the thin gold films in our case) driven by the equilibrium deformation $\Delta \bar{d}_{M}(t)=\alpha_{L}\left[T_{L}(t)-T_{0}\right] d_{M}$, with $\alpha_{L} \simeq 1.5 \times 10^{-5} \mathrm{~K}^{-1}$ the linear coefficient of thermal expansion in gold [41]. Therefore, to determine $\Delta d_{M}$ one has to solve for the following ordinary differential equation:

$$
\frac{d^{2}}{d t^{2}} \Delta d_{M}+\frac{2}{\tau_{\mathrm{osc}}} \frac{d}{d t} \Delta d_{M}+\frac{4 \pi^{2}}{T_{\mathrm{osc}}^{2}} \Delta d_{M}=\frac{4 \pi^{2}}{T_{\mathrm{osc}}^{2}} \Delta \bar{d}_{M}(t),
$$

where $T_{\text {osc }}$ is the period of the acoustic oscillation and $\tau_{\text {osc }}$ its damping time. Typically these parameters ought to be estimated by fitting the differential transmission measurements on the long time scale, as detailed in our analysis of the experimental data reported in Sec. III (Fig. 5). However, if $T_{\text {osc }}$ becomes comparable to the heating time of the lattice (of the order of a few ps), and in the presence of strong damping (i.e., $\tau_{\mathrm{osc}}$ comparable to $T_{\mathrm{osc}}$, as in our case), the oscillation becomes very weak (see, e.g., Ref. [39]) and the $\Delta d_{M}(t)$ can be approximated by the equilibrium deformation $\Delta \bar{d}_{M}(t)$. Such an approximation allows one to avoid the employment of the two fitting parameters $T_{\text {osc }}$ and $\tau_{\text {osc }}$, at the expense of a negligible error in the disentanglement of the different contributions to the optical nonlinearity (due to the omission of the weak oscillatory phenomenon).

Figure 8 shows the real and imaginary part of each separate contribution to $\Delta \epsilon$ for the excitation conditions of our experiments. The contribution arising from nonthermalized carriers [Fig. 8(a)] is spectrally much broader than the contribution arising from thermalized ones [Fig. 8(b)]. Actually, nonthermalized carriers are generated in a large interval of electron energies, from $E_{F}-h v_{P}$ to $E_{F}+h v_{P}$, $E_{F}$ being the Fermi energy of gold and $h v_{P}$ the pump photon energy [30]. Conversely, thermalized carriers mostly affect the permittivity of the metal for optical transitions involving electronic energies close to $E_{F}$, in view of the smearing of the Fermi-Dirac distribution due to the heating of thermalized carriers. Therefore $\Delta \epsilon_{T}$ is peaked close to the L-point and X-point IB optical transitions. Both the two contributions $\Delta \epsilon_{N T}$ and $\Delta \epsilon_{T}$ decrease to zero for long time delays. Regarding the lattice contributions, $\Delta \epsilon_{L 1}$ and $\Delta \epsilon_{L 2}$ grow slowly though monotonically in time since the lattice temperature increases on a time scale of a few ps. Another important difference between the four contributions to the $\Delta \epsilon$ is the following one: far from IB transitions $\Delta \epsilon_{T}$ is dominated by its real part exhibiting normal dispersion, whereas for the $\Delta \epsilon_{N T}$ both real and imaginary parts are relevant in a broad range of wavelengths with nontrivial dispersive behavior. The $\Delta \epsilon_{L 1}$ is dominated by its imaginary part whereas $\Delta \epsilon_{L 2}$ is dominated by its real part and both the two exhibit anomalous dispersion. In the visible, this latter contribution is the weakest among the four $\Delta \epsilon$ and typically it is neglected, but in the NIR of our experiment it turns out to be much stronger (because of the anomalous dispersion) and for long time delays it becomes much larger than $\Delta \epsilon_{N T, T}$ and of the order of $\Delta \epsilon_{L 1}$. Finally, note that at around 500 fs time delay [dashed curves in Figs. 8(a)-8(d)] all the four $\Delta \epsilon$ are approximately of the same order (in modulus) and thus compete with each other for the establishment of the resulting Kerr nonlinearity. 

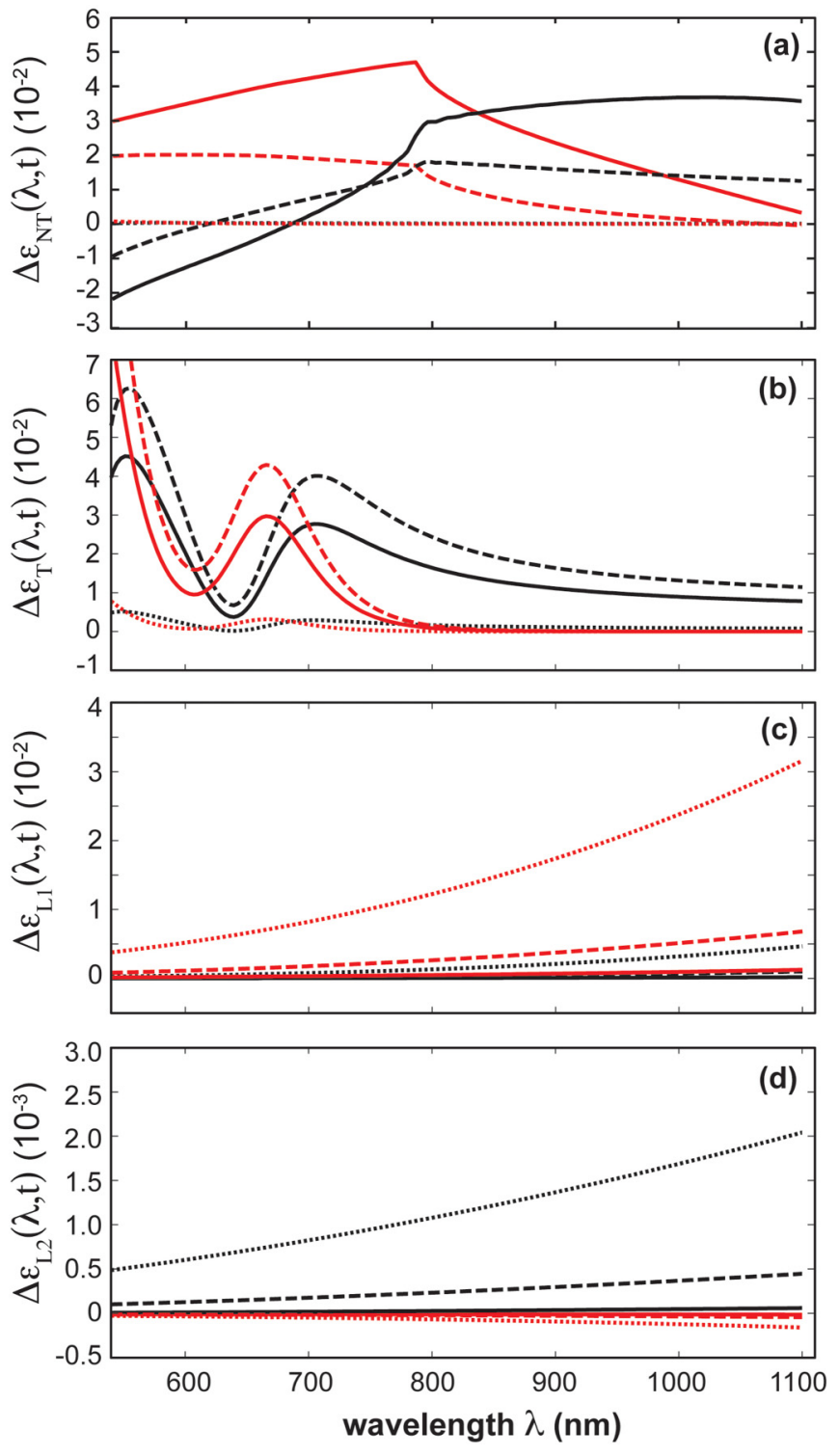

FIG. 8. (Color online) Real part (black curves) and imaginary part (red curves) of the dielectric function modulations of gold due to (a) nonthermalized carriers, (b) thermalized carriers, and (c) and (d) the two lattice mechanisms, at different time delays: $t=150 \mathrm{fs}$ (solid line), $t=500 \mathrm{fs}$ (dashed line) and $t=4 \mathrm{ps}$ (dotted line). We considered a pump-pulse power absorbed in the unit volume $P_{a}(t)=$ $P_{0} \exp \left[-4 \ln (2) t^{2} / \tau_{P}\right]$ with $P_{0}=6.5 \times 10^{13} \mathrm{~W} / \mathrm{cm}^{3}$, corresponding to the excitation conditions used in the experiments of Fig. 3(a).

Our results clearly indicate a complex scenario for the Kerr nonlinearity of gold, which combined with the interferential nature of the linear optical response of the MDBF prevents any possibility for a disentanglement of the rich spectral features observed in the $\Delta T / T$ experimental map of Fig. 3(a), without an accurate modeling of all the physical processes involved.

\section{Numerical simulation of pump-probe experiments}

To model pump-probe experiments, we computed the theoretical differential transmission of the MDBF as $\Delta T / T=$ $T^{\prime}(\lambda, t) / T(\lambda)-1, T(\lambda)$ being the linear transmittance of the unperturbed MDBF and $T^{\prime}(\lambda, t)$ the MDBF transmittance for gold layers with permittivity $\epsilon_{A u}(\lambda)+\Delta \epsilon(\lambda, t)$, with $\Delta \epsilon(\lambda, t)$ the sum of all the four contributions detailed above. Results are reported in Fig. 3(b) and calculated $\Delta T / T$ dynamics at some selected wavelengths are shown in Fig. 4 (dashed blue curves).

Comparison between Figs. 3(a) and 3(b) and between the experimental and theoretical cross sections of Fig. 4 indicates a good quantitative agreement between pump-probe experiments and numerical simulations. It is worth noting that contrary to previous studies on metallic multilayers [17-20], our simulations are performed with no fitting parameters. This confirms the validity of the model developed for thin films but in a broader wavelength range, that is far from the IB optical transitions of gold. In particular, the model quantitatively reproduces several features, including (i) the spectral position of positive and negative peaks, (ii) the relative intensity of positive and negative peaks, (iii) the slight redshift of the peaks with increasing time delay (more evident for the negative ones), and (iv) the absolute value of the $\Delta T / T$ at long time delays. Only a slight deviation is observed in the initial part of the dynamics where the numerical simulations predict a shorter duration for the dynamics of the negative lobes of the map (see below for a discussion of this anomaly).

\section{DISCUSSION}

\section{A. Disentanglement of Kerr nonlinearity}

The numerical model can be exploited to investigate the origin of the different spectral features observed in the $\Delta T / T$ experimental map and eventually disentangle the different contributions to the transient optical response of the MDBF due to the different degrees of freedom illustrated in Sec. IV.

To this purpose, we computed the $\Delta T / T$ maps corresponding to individual contributions to the Kerr nonlinearity arising from nonthermalized carriers only, from thermalized carriers only, and from the lattice only. These are shown in Fig. 9, and their comparison reveals that (i) the negative peaks at short time delays are dominated by nonthermalized carriers [Fig. 9(a)], with a minor contribution from thermalized ones [Fig. 9(b)]; (ii) the positive peaks at short time delays are mainly ascribable to thermalized carriers, but also nonthermalized carriers are involved; (iii) negative peaks at long time delays are due to lattice heating [Figs. 9(c) and 9(d)] and the Drude damping increase prevails over the plasma frequency decrease, even though the two mechanisms give rise to different effects. More precisely, the first mechanism always provides a negative contribution to the $\Delta T / T$, whereas the second one provides both positive and negative modulation. This can be understood if one considers that the $\Delta \epsilon_{L 1}$ is mainly imaginary [Fig. 8(c)] whereas the $\Delta \epsilon_{L 2}$ is mainly real and positive [Fig. 8(d)]. Therefore, it is expected that the $\Delta \epsilon_{L 1}$ introduces a broadening of the transmission peaks of the MDBF whereas the $\Delta \epsilon_{L 2}$ causes a redshift of the peaks with subsequent derivative behavior in the $\Delta T / T$. Finally, it is worthy of note that the thermal expansion inherent in lattice heating (and underlying the $\Delta \epsilon_{L 2}$ ) could give rise to another contribution to the $\Delta T / T$ induced by modulation of the Bragg effect because of variation of metal layer thickness, that we have omitted. However, we numerically estimated this contribution and it turned out to be an order of magnitude 


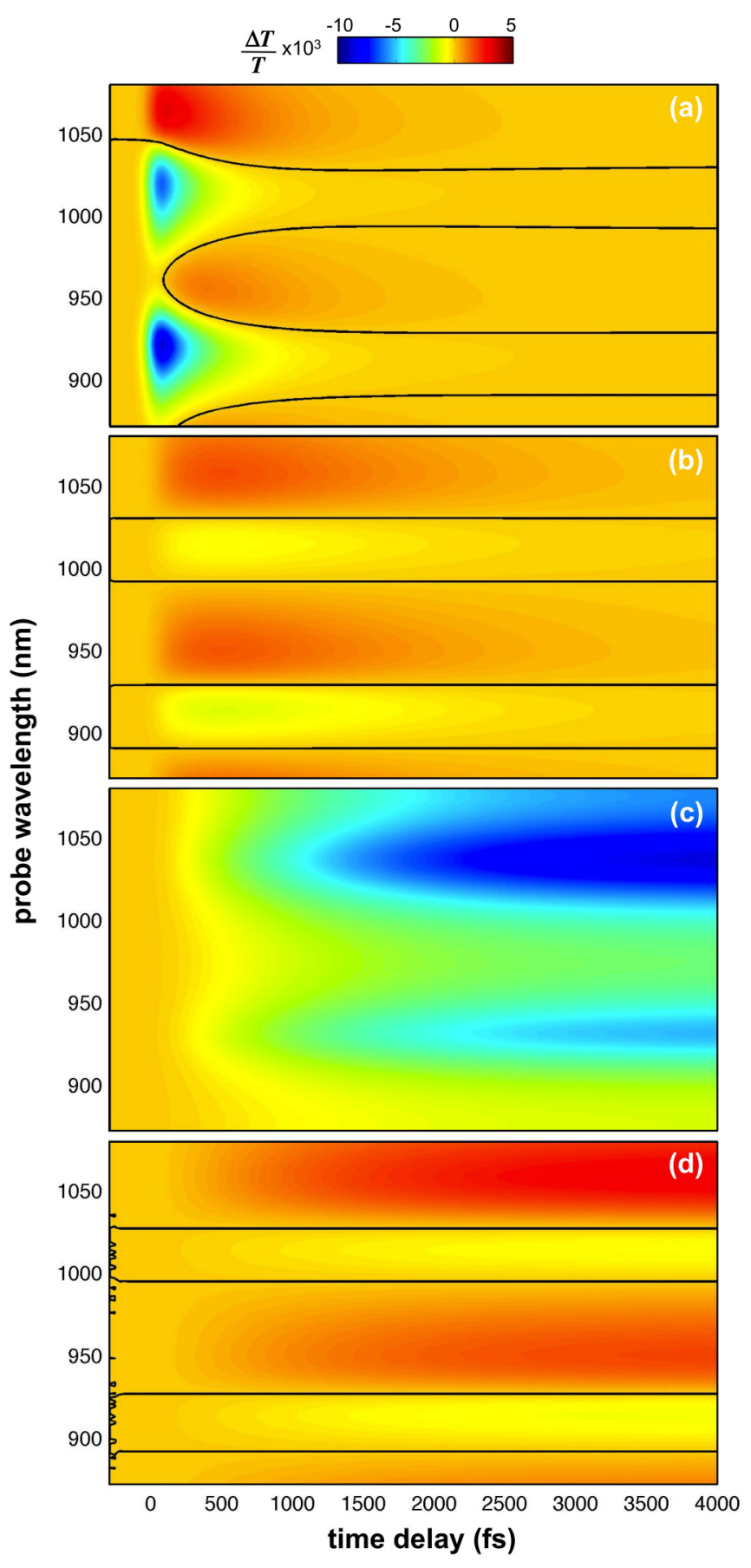

FIG. 9. (Color online) Different contributions to the total $\Delta T / T$ of Fig. 3 arising from (a) nonthermalized electrons, (b) thermalized electrons, (c) Drude damping increase, and (d) plasma frequency decrease $(10 \times$ magnified for better comparison). Superposed black line represents the isosbestic of each individual map.

lower as compared to the $\Delta T / T$ modulation induced by the plasma frequency decrease. About the $\Delta \epsilon_{L 2}$, it is worth remembering also that we have considered an adiabatic approximation of the actual dynamics of the plasma frequency mechanism (cf. Sec. IV B 2), allowing a good estimation of the contribution to the $\Delta T / T$ on this time scale provided that the acoustic oscillations are negligible. Otherwise an overshoot at the end of electron-lattice thermalization and long-living oscillations can give rise to a much higher contribution to the optical nonlinearity from the plasma frequency modulation induced by lattice heating. The fact that in the MDBF such oscillatory dynamics is almost absent (cf. end of Sec. III) can be possibly explained according to the following arguments: (i) a dephasing mechanism induced by inhomogeneities between the five oscillators, having possibly different resonance frequencies mostly because of their different mechanical boundary conditions [cf. sketch of the MDBF in Fig. 1(a)]the dephasing induced by inhomogeneities is recognized as the fundamental cause of oscillation damping in ensembles of metallic nanoparticles (much stronger than the energy transfer to the surrounding medium) [39]; (ii) mechanical coupling between the oscillators (due to the mechanical contact provided by the thin glass spacers), causing splitting of the fundamental acoustic resonance and thus further dephasing. Also, note the shortness of the oscillation period ( $\sim 7 \mathrm{ps})$ as compared to other papers on metallic film structures where the oscillation period was of a few tens of ps (because of the thickness of the films). Therefore, in our case the heating time (of the order of a few ps) ceases to be almost instantaneous and the lattice is heated during a significant fraction of the oscillation cycle, with subsequent onset of a washout mechanism [39]. An investigation of these mechanisms is beyond the scope of the present paper, but both experiments and general arguments based on previously published literature indicate that acoustic oscillations are of minor relevance in the nonlinear optical response of gold-based MDBFs.

The disentanglement of all the different contributions to the $\Delta T / T$ also allows one to identify the origin of the slight anomaly observed on the shortest time scale, where it is seen that the negative peaks in the experimental map [Fig. 3(a)] evolve more slowly as compared to numerical simulations [compare in particular the experimental (solid curve) and theoretical (dashed curve) map cross sections in Fig. 4(a)]. Since the negative peaks turned out to be dominated by the $\Delta \epsilon_{N T}$, the anomaly can be ascribed to an overestimation (by about $50 \%$ ) of the decay rate of nonthermalized carriers retrieved by the ETTM model. This discrepancy is much higher than that observed in the visible (cf. Fig. 2 in Ref. [24]), and can be attributed to the approximations introduced in the Boltzmann equations to analytically derive Eq. (1), and in particular the hypothesis (based on Fermi liquid theory) that the electronelectron scattering rate scales quadratically with the electron energy (measured from the Fermi energy), without any specific dependence on the actual electronic states of gold (apart from a constant multiplier). Evidently this approximation is too rough to allow quantitative agreement for transitions involving electronic states far from the Fermi energy, which is the case of optical transitions in the NIR of our experiments. Apart from this obvious limitation, the ETTM is capable of reproducing almost all the features observed on the sub-ps time scale in the $\Delta T / T$ map, including the sign change and the ultrafast oscillations [compare the experimental (solid curve) and theoretical (dashed curve) map cross sections in Figs. 4(b) and 4(c)].

\section{B. Evaluation of optical-modulation capability}

To appreciate the advantages offered by the multilayer configuration as compared to the single metallic layer, we performed pump-probe experiments and numerical simulations 

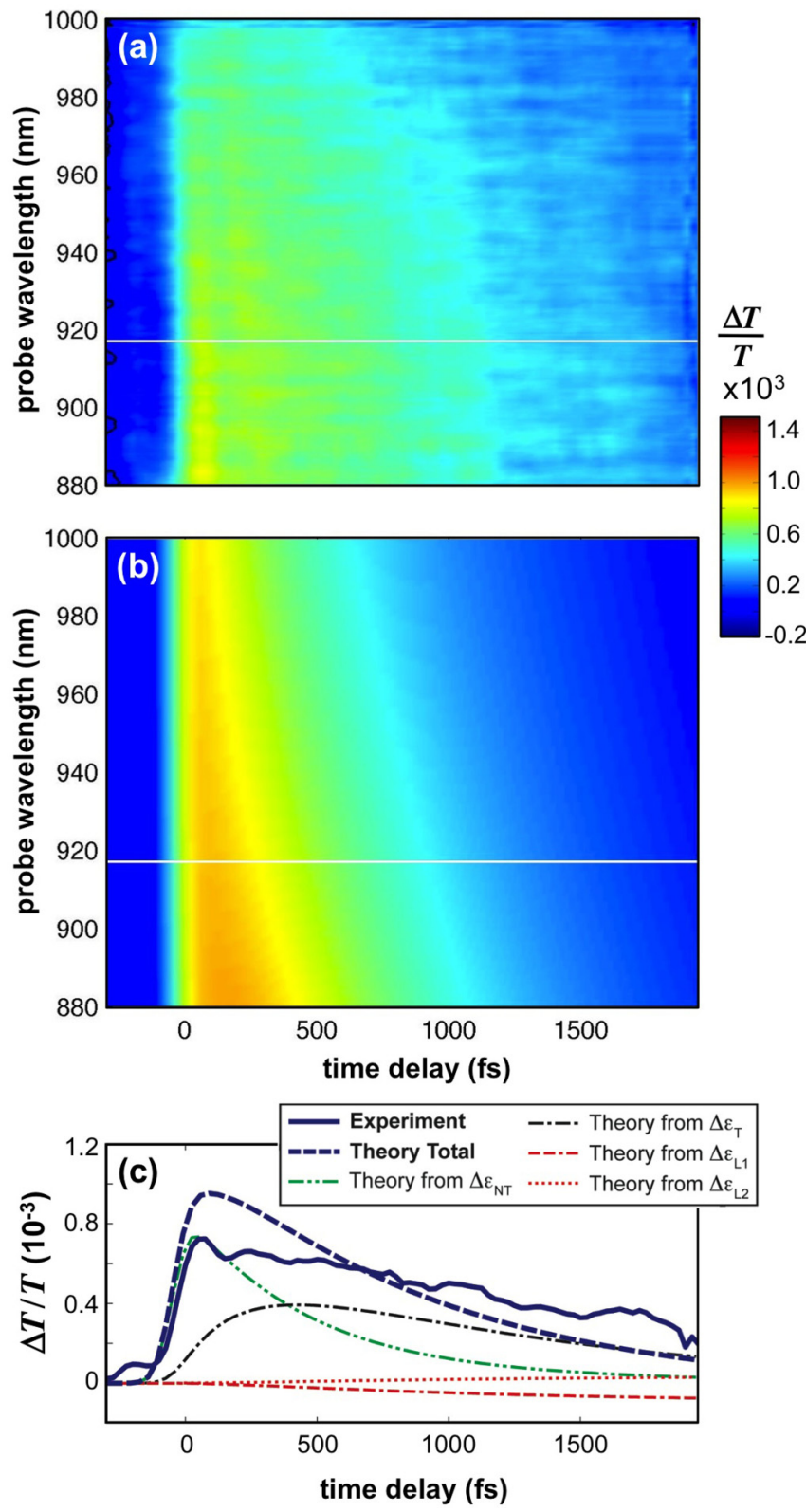

FIG. 10. (Color online) Differential transmission map of a $30 \mathrm{~nm}$ thin gold film on silica under $310 \mu \mathrm{J} / \mathrm{cm}^{2}$ incident fluence: (a) experimental data, (b) numerical simulation. Panel (c) shows cross sections of the $\Delta T / T$ maps at $915 \mathrm{~nm}$ : experimental data (solid line) are quantitatively compared to numerical simulations showing the total $\Delta T / T$ (dashed line) and the four different contributions to the $\Delta T / T$ arising from nonthermalized (dash-double-dot line) and thermalized (dash-dot line) electrons, and from the lattice, including the effect related to the Drude damping increase (double-dash-dot line) and to the plasma frequency decrease (dotted line).

on the $30 \mathrm{~nm}$ thin gold film described in Sec. II. We adopted the same experimental setup and identical pumping conditions as those employed for the MDBF.

The experimental $\Delta T / T$ is shown in Fig. 10(a) and the theoretical map in Fig. 10(b). The good quantitative agreement between experiments and simulations corroborates the analysis above reported for the MDBF. As expected, the $\Delta T / T$ of the film exhibits an almost uniform spectrum with no relevant features, despite the complex spectral behavior of the four different $\Delta \epsilon$ terms detailed in Sec. IV (Fig. 8). Most importantly, note that, at around $915 \mathrm{~nm}$, the maximum $\Delta T / T$ achieved from the single layer is about $+0.1 \%$ [Fig. 10(c)] against the peak value of $-0.8 \%$ achieved from the MDBF [Fig. 4(a)]. This improvement is only partially due to the higher pump energy deposited in the sample when pumping close to an absorption peak of the MDBF, as indicated by the numerical integration of the ETTM Eq. (1) showing a peak electronic temperature increase which is about two times higher than in the film (Fig. 7). Therefore, the main reason for the more pronounced modulation offered by the multilayer configuration is in the complex spectral modification of the MDBF transmission peaks induced by the $\Delta \epsilon$. These peaks shift and broaden as a consequence of the Kerr nonlinearity, and thus give rise to larger variations of the probe transmittance at selected wavelengths. Most importantly, since the transmission of the MDBF at around $915 \mathrm{~nm}(T \sim 0.25)$ is about 5 times higher as compared to the transmission of the film $(T \sim 0.05)$, the comparison in terms of $\Delta T$ (which is a more interesting figure of merit in the perspective of optical-switching) reads as follows: $\Delta T \simeq-0.2 \%$ in the MDBF against $\Delta T \simeq+0.005 \%$ in the single film. The MDBF outperforms the single film by a factor of 40

It is worth noting that the pump fluence of our experiments was kept low to achieve perturbative excitation of the system, allowing accurate modeling of all the transient dynamical processes involved in the Kerr nonlinearity of gold and thus accurate disentanglement of each individual contributions to the transient optical spectrum. Actually, when the electronic temperature increase $\Delta T_{e}$ exceeds a few hundred $\mathrm{K}$, the $\Delta \epsilon_{T}$ is no longer linearly proportional to the $\Delta T_{e}$ [8] giving rise to spectral deformations that can in principle modify the position of the peaks and dips of the $\Delta T / T$ map. For the MDBF pumped at $835 \mathrm{~nm}$ this limit is achieved for an incident pump fluence of about $500 \mu \mathrm{J} / \mathrm{cm}^{2}$. Also, the modeling of nonthermalized carrier dynamics, responsible for the $\Delta \epsilon_{N T}$, has never been validated in the nonperturbative regime. However, pump-probe experiments at higher pump power levels (the main results are shown in Fig. 11) indicate that the $\Delta T / T$ scales linearly with the pump fluence even beyond the perturbative excitation limit. It is thus expected that nonperturbative excitation with a few tens of $\mathrm{mJ} / \mathrm{cm}^{2}$ can provide sizable optical modulation, with $\Delta T$ of a few tens $\%$, in selected transmission windows in the NIR, a possibility which is totally prevented by using a single metal layer of gold.

The main limitation of the MDBF is represented by the long-living contribution to the transmission modulation arising from lattice heating [Figs. 9(c)-9(d); see also circles in Fig. 11], which is known to decay to zero in hundreds ps by the phonon-phonon interaction process governing the heat transfer between the metal lattice and the environment (see, e.g., $[37,39]$ and references therein). This poses an upper limit to the modulation speed of a MDBF device, which is of the order of $10 \mathrm{GHz}$. Even though this is a general issue of any metal-based optical system, in the multilayer configuration it is somehow enhanced as compared to the single metal layer (cf. Figs. 3 and 10). Such an increase in the long-living response stems from the same resonant features which are primarily responsible for the enhanced modulation observed on the sub-ps time 


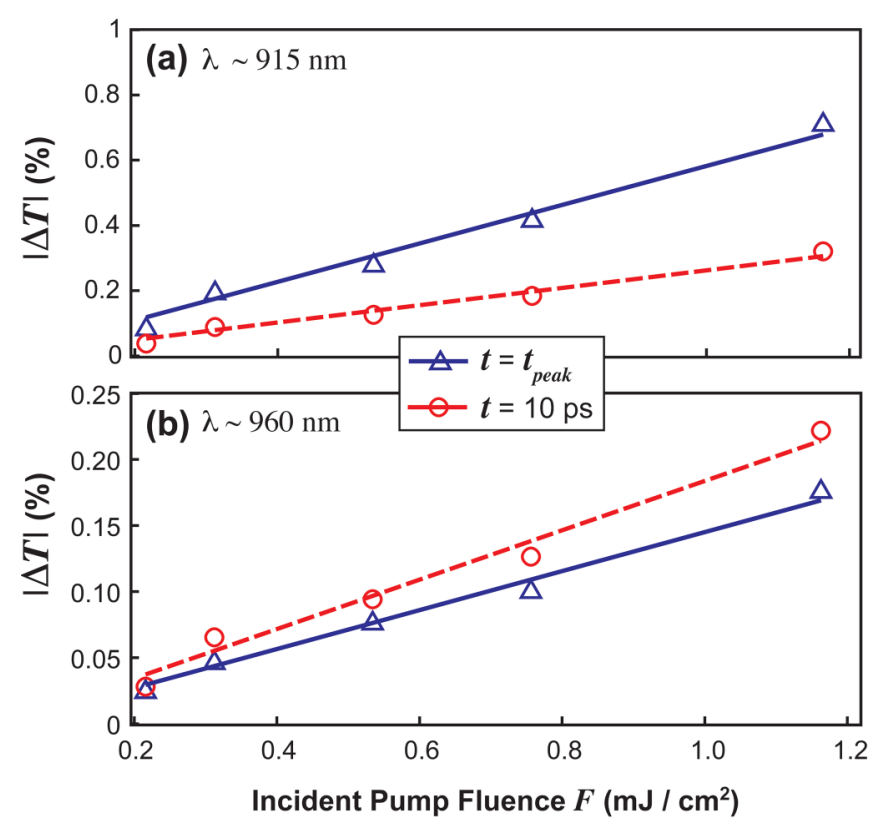

FIG. 11. (Color online) Peak (absolute value) of the $\Delta T$ (triangles) achieved from the MDBF at (a) $\sim 915 \mathrm{~nm}$ and (b) $\sim 960 \mathrm{~nm}$ probe wavelengths as a function of the incident pump fluence. Long-living value of the $\Delta T$ (measured at $10 \mathrm{ps}$ time delay) is also shown (circles). Straight lines represent linear interpolation of the experimental points.

scale: the increase of the imaginary part of gold permittivity $\Delta \epsilon_{L 1}$ due to lattice heating causes the transmission peaks to broaden primarily because of increased absorption at selected wavelengths, with subsequent formation of negative peaks (i.e., decreased transmission) in the transient optical response. In the single gold layer, having an almost flat linear transmission in the NIR, the $\Delta \epsilon_{L 1}$ gives rise to a nonresonant and thus very weak negative $\Delta T / T$ [which in the present case of a 30-nm-thick layer is comparable to the positive contribution originated from the weaker $\Delta \epsilon_{L 2}$; compare the double-dash-dot line and dotted line in Fig. 10(c)]. Therefore, after the dynamics of nonthermalized and thermalized carriers is completely exhausted ( $\sim 10 \mathrm{ps})$, a spectrally flat and very weak negative $\Delta T / T$ is achieved in the single film.

\section{CONCLUSION}

We investigated the transient optical response of a MDBF made of 15-nm-thick gold layers and 260-nm-thick silica spacers. The experimental analysis was performed by ultrafast broad-band differential transmission spectroscopy. Numerical modeling of the optical nonlinearity in the metal allowed us to disentangle the rich spectral features observed in the NIR. We found that nonthermalized carriers play a key role in the onset of differential transmission peaks in the sub-ps time scale. This is basically due to the fact that nonthermalized carriers are generated by plasmon dephasing in a broad range of energies, whereas thermalized carriers mostly contribute to the optical response close to the IB transition edge and thus dominate Kerr nonlinearity only in the visible. Also, the lattice heating is responsible for a spectrally shaped transient response at long time delays.

Our results can support further theoretical developments on the formulation of a noninstantaneous Kerr nonlinearity of gold with respect to previous papers [8-10], by possibly including the contribution from nonthermalized carriers as well as from the lattice heating. We also provided a quantitative clear-cut comparison of the optical-modulation capability of the MDBF with respect to a single metal film under the same pumping conditions. The gold MDBF can provide up to 40 times improvement of the modulation capability as compared to a single gold layer, within suitable transmission bands. This makes the scaling to sizable modulations (i.e., $\Delta T$ of some tens \%) feasible under nonperturbative optical excitation with tens of $\mathrm{mJ} / \mathrm{cm}^{2}$ fluence when pumping the MDBF at peak absorption resonances in the NIR. However, the same interferential mechanism responsible for the enhancement of the ultrafast nonlinear response also enhances the long-living contribution arising from lattice heating, thus posing some limitations in terms of recovery time.

\section{ACKNOWLEDGMENTS}

This work was supported by Fondazione Cariplo through the projects "Engineering of Optical Nonlinearities in Plasmonic Metamaterials" (Grant No. 2010-0595) and "New Frontiers in Plasmonic Nanosensing" (Grant No. 2011-0338). G.C. acknowledges support by the EC under Graphene Flagship (Contract No. CNECT-ICT-604391). E.A., D.P., and R.B. acknowledge Monica Bollani for the SEM images.
[1] S. Lal, S. Link, and N. J. Halas, Nat. Photon. 1, 641 (2007).

[2] J. A. Schuller, E. S. Barnard, W. Cai, Y. Jun, J. S. White, and M. 1. Brongersma, Nat. Mater. 9, 193 (2010).

[3] H. Raether, Surface Plasmons, 1st ed. (Springer-Verlag, Berlin, 1986).

[4] M. Kauranen and A. V. Zayats, Nat. Photon. 6, 737 (2012).

[5] A. Husakou, S.-J. Im, and J. Herrmann, Phys. Rev. A 83, 043839 (2011).

[6] K. F. MacDonald, Z. L. Samson, M. I. Stockman, and N. I. Zheludev, Nat. Photon. 3, 55 (2009).

[7] M. I. Stockman, New J. Phys. 10, 025031 (2008).

[8] M. Conforti and G. Della Valle, Phys. Rev. B. 85, 245423 (2012).
[9] A. Marini, M. Conforti, G. Della Valle, H. Lee, T. X. Tran, W. Chang, M. A. Schmidt, S. Longhi, P. S. J. Russell, and F. Biancalana, New J. Phys. 15, 013033 (2013).

[10] H. Baida, D. Mongin, D. Christofilos, G. Bachelier, A. Crut, P. Maioli, N. Del Fatti, and F. Vallée, Phys. Rev. Lett. 107, 057402 (2011).

[11] D. D. Smith, Y. Yoon, R. W. Boyd, J. K. Campbell, L. A. Baker, R. M. Crooks, and M. George, J. Appl. Phys. 86, 6200 (1999).

[12] T. K. Lee, A. D. Bristow, J. Hubner, and H. M. van Driel, J. Opt. Soc. Am. B 23, 2142 (2006).

[13] N. Rotenberg, A. D. Bristow, M. Pfeiffer, M. Betz, and H. M. van Driel, Phys. Rev. B 75, 155426 (2007). 
[14] R. W. Boyd, in Nonlinear Optics, 2nd ed. (Academic, New York, 2003), p. 212, Chap. 4.

[15] M. Ren, B. Jia, J.-Y. Ou, E. Plum, J. Zhang, K. F. MacDonald, A. E. Nikolaenko, J. Xu, M. Gu, and N. I. Zheludev, Adv. Mater. 23, 5540 (2011).

[16] G. A. Wurtz, R. Pollard, W. Hendren, G. P. Wiederrecht, D. J. Gosztola, V. A. Podolskiy, and A. V. Zayats, Nat. Nanotechnol. 6, 107 (2011).

[17] R. S. Bennink, Y.-K. Yoon, R. W. Boyd, and J. E. Sipe, Opt. Lett. 24, 1416 (1999).

[18] N. N. Lepeshkin, A. Schweinsberg, G. Piredda, R. S. Bennink, and R. W. Boyd, Phys. Rev. Lett. 93, 123902 (2004).

[19] T. Ergin, T. Benkert, H. Giessen, and M. Lippitz, Phys. Rev. B 79, 245134 (2009).

[20] P. Farah, N. Gibbons, F. M. Huang, and J. J. Baumberg, Phys. Rev. B 84, 125442 (2011).

[21] A. Husakou and J. Herrmann, Phys. Rev. Lett. 99, 127402 (2007).

[22] D. T. Owens, C. Fuentes-Hernandez, J. M. Hales, J. W. Perry, and B. Kippele, Opt. Express 18, 19101 (2010).

[23] E. Carpene, Phys. Rev. B 74, 024301 (2006).

[24] G. Della Valle, M. Conforti, S. Longhi, G. Cerullo, and D. Brida, Phys. Rev. B 86, 155139 (2012).

[25] S. Brivio, C. Magen, A. A. Sidorenko, D. Petti, M. Cantoni, M. Finazzi, F. Ciccacci, R. De Renzi, M. Varela, S. Picozzi, and R. Bertacco, Phys. Rev. B 81, 094410 (2010).

[26] P. Yeh, in Optical Waves in Layered Media, 1st ed. (Wiley, New York, 1988), pp. 102-112, Chap. 5.

[27] P. G. Etchegoin, E. C. Le Ru, and M. Meyer, J. Chem. Phys. 125, 164705 (2006).

[28] I. H. Maltison, J. Opt. Soc. Am. 55, 1205 (1965).
[29] D. Polli, L. Luer, and G. Cerullo, Rev. Sci. Instrum. 78, 103108 (2007).

[30] C.-K. Sun, F. Vallée, L. H. Acioli, E. P. Ippen, and J. G. Fujimoto, Phys. Rev. B 50, 15337 (1994).

[31] R. H. M. Groeneveld, R. Sprik, and A. Lagendijk, Phys. Rev. B 51, 11433 (1995).

[32] The ETTM comprises two response functions [23,24], $H_{e e}(t)$, corresponding to the $H(t)$ function considered here, and $H_{e p}(t)$, taking into account the energy transfer between nonthermalized electrons and lattice and thus entering the second Eq. (1). Since in gold this latter response is two orders of magnitude weaker than the former one (see, e.g., Ref. [8]), we have neglected the $H_{e p}(t)$.

[33] P. Winsemius, M. Guerrisi, and R. Rosei, Phys. Rev. B 12, 4570 (1975).

[34] M. Guerrisi, R. Rosei, and P. Winsemius, Phys. Rev. B 12, 557 (1975).

[35] F. Masia, W. Langbein, and P. Borri, Phys. Rev. B 85, 235403 (2012).

[36] R. T. Beach and R. W. Christy, Phys. Rev. B 16, 5277 (1977).

[37] N. Del Fatti, C. Voisin, D. Christofilos, F. Vallée, and C. Flytzanis, J. Phys. Chem. A 104, 4321 (2000).

[38] J. H. Hodak, I. Martini, and G. V. Hartland, J. Chem. Phys. 108, 9210 (1998).

[39] J. H. Hodak, A. Henglein, and G. V. Hartland, J. Phys. Chem. B 104, 9954 (2000).

[40] G. V. Hartland, Chem. Rev. 111, 3858 (2011).

[41] N. W. Ashcroft and N. D. Mermin, in Solid State Physics, 1st ed. (Harcourt College Publishers, Fort Worth, 1976), p. 494, Chap. 25. 